\title{
MEJORAMIENTO DE LA PRODUCTIVIDAD EN UNA INDUSTRIA MADERERA USANDO INCENTIVO REMUNERATIVO
}

\author{
PRODUCTIVITY IMPROVEMENT IN A SAWNWOOD MILL USING SALARY \\ INCENTIVE
}

\author{
Patricio Pino-Pinochet ${ }^{1}$, Mauricio Ponce-Donoso ${ }^{1, \wedge}{ }^{\text {, Carmen Avilés-Palacios }}{ }^{2}$, \\ Óscar Vallejos-Barra ${ }^{1}$
}

\begin{abstract}
RESUMEN
Se estudió el efecto en la productividad de la elaboración de blanks a partir de la implementación de un modelo de incentivo salarial del tipo Improshare, dirigido al personal de una empresa chilena de remanufacturado de maderera que produce molduras y marcos de puerta (ayudante, operador medio y operador máster), para lo cual se compararon dos periodos de tiempo, antes (19 meses) y después (21 meses) de la implementación de la política de incentivo. Los resultados mostraron diferencias estadísticamente significativas en la productividad lograda entre periodos que superó el $10 \%$. Se midió el beneficio promedio de la remuneración de los trabajadores, que llegó a más del 10\% y el ahorro de la empresa llegó al 4,6\% de la unidad monetaria definida, sin que se haya visto afectada la tasa de accidentabilidad. Lo anterior refuerza lo señalado por diferentes autores que concuerdan que este tipo de herramientas contribuye a mejorar la productividad del proceso e incrementar el salario del trabajador. Su implementación como política salarial en la industria maderera, aporta significativamente a los desafíos de una organización por mantenerse competitiva, así como mejorar la remuneración del trabajador en base a una relación de beneficio mutuo con la empresa.
\end{abstract}

Palabras Clave: Improshare, incentivo laboral, industria remanufacturera, pino radiata, política de remuneraciones.

\begin{abstract}
We studied the effect on productivity resulting in the blanks elaboration from the implementation of a salary incentive model of Improshare type for staff working group in a sawnwood mill company producer remanufacturing moldings and door frames (assistant, operator and operator master), for which two time periods were compared, before (19 months) and after (21 months) of incentive policy implementation. The results show statistically significant differences in the productivity between periods, of more than $10 \%$. The average benefit of the workers compensation was measured, it reached more than $10 \%$ and the savings to the company reached $4,6 \%$ of defined monetary unit, without affecting the accident rate. This reinforces the point made by different authors that this kind of tool improves the productivity of the process and increases wages. Its implementation as compensation policy in the lumber industry contributes significantly to the challenges that an organization faces to remain competitive and improve workers compensation based on a mutually beneficial relationship with the company.
\end{abstract}

Keywords: Improshare, radiata pine, remanufacture lumber mill, salary policy, wage incentive.

\footnotetext{
${ }^{1}$ Facultad de Ciencias Forestales. Universidad de Talca. Avda. Lircay s/n, Talca . Chile.

${ }^{2}$ Escuela Técnica Superior de Ingeniero de Montes. Universidad Politécnica de Madrid. Ciudad Universitaria s/n. 280040. Madrid. España.

^Autor para correspondencia: mponce@utalca.cl

Recibido: 10.12. 2013 Aceptado: 31.03. 2014
} 


\section{INTRODUCCIÓN}

Los recursos humanos se consideran como elementos esenciales en una organización, dada su capacidad para generar ventajas competitivas que la hagan diferente a otras. El talento humano según Infante (2008) es un motor que permite a la organización el logro de los planes y objetivos diseñados y es un factor fundamental en las decisiones para un adecuada gestión de las operaciones productivas y el desarrollo de la organización (Boothby et al. 2010, Buller y Mc Evoy 2012). Es necesario, por tanto, conseguir que las personas que trabajan en una organización estén motivadas, ya cumplen un rol estratégico en cualquier organización y son responsables de transformar insumos en productos o servicios.

La motivación de los empleados es una de las políticas utilizadas por la dirección para conseguir un incremento de competitividad. Existe un amplio abanico de métodos que persiguen motivar al personal, siendo los más relevantes un salario adecuado al trabajo desarrollado por el empleado (Rynes et al. 2004); las recompensas, entendidas como aquellas herramientas de gestión que contribuyen a la mejora de la eficiencia de la organización, influyendo en el comportamiento individual o de grupo (Kamalian et al. 2010); el liderazgo y el apoderamiento o empowerment, el cual beneficia a la organización y genera el sentimiento de orgullo y pertenencia entre los empleados (Yazdani et al. 2011); y la confianza, entendida como la percepción que tienen los demás sobre uno mismo (Annamalai 2010). Rynes et al. (2004) establecen que la compensación monetaria es la única forma para generar la motivación en el trabajador, aunque señalan que no hay evidencias que el dinero sea el único motivador para la mayoría de las personas. Por ello, los gerentes debieran considerar herramientas de tipo financieras como no monetarias, para atraer, motivar y retener trabajadores. Sin embargo, los autores apuntan que el uso de estas herramientas debe ser evaluado en cada caso, considerando tanto variables situacionales como individuales.

Un sistema de incentivos es un conjunto de reglas o principios que permiten desarrollar métodos para lograr la participación de los trabajadores en la producción de bienes (Caso 2003). El mismo autor lo define también como un algoritmo que relaciona la cantidad a pagar con el resultado de la actuación que da lugar al incentivo. Según sea el algoritmo, estos sistemas pueden ser de tipo progresivo, regresivo y lineal (proporcionales). Los tres sistemas tienen en común que el incentivo parte de un resultado exigible y tiene un máximo alcanzable al llegar al resultado óptimo (Caso 2003). El sistema progresivo supone que el esfuerzo para conseguir un resultado es tanto mayor cuanto más cercano esté al resultado óptimo y, por tanto debe de remunerarse más (Caso 2003). Dentro de esta categoría los hay de tipo individuales, entre ellos por producción, volumen, e inferior al pleno ahorro sistemas Hasley y Rowan (Caso 2003); y los de tipo grupal, como Scalon, Rucker e Improshare (Meyers 2000) los que, en todo caso, deben estar relacionados con la modificación de las conductas identificadas como problemas (Kohn 1993).

La incorporación de la productividad como elemento de salario da como resultado remuneraciones variables, lo que atentaría contra la certidumbre y estabilidad del mismo, efecto que es evidente en una perspectiva de mediano y largo plazo. Con el pago variable las ganancias suben y bajan con la medición del desempeño, cuya fluctuación del pago variable hace que esta tipología de programas sean atractivos para la administración. Sin embargo, lo que en su momento es incremento o reajuste de remuneraciones, al cabo de los años pasan a incorporarse como parte estructural del salario que, de fijo pasa a ser progresivamente variable (Vergara 1998). Parte de los costos fijos de la mano de obra se convierten en costos variables, que se reducen cuando el desempeño disminuye (Robbins 2004).

Según Caso (2003) los incentivos corresponden a la parte variable del salario o reconocimiento que premia un resultado superior al exigible. La obtención de este resultado es voluntaria, por lo que puede ser incentivado y debiera ser pactado. La responsabilidad de la empresa se resume en conseguir este pacto y poner los medios para su cumplimiento. Segura (2011) señala que la mayoría de los trabajadores reconocen cumplir con las metas de producción con holgura, pero declaran que no existen estímulos para el mejoramiento de la productividad, por lo que recomienda diseñar una política salarial compatible con los incentivos del trabajador para un trabajo productivo y seguro, concordante con los resultados planteados. 
Milkovich et al. (2011) indican que los escenarios de incentivos constituyen componentes financieros de compensación para los empleados, dado que el pago incrementa el desempeño y tiende a ser usado por las organizaciones con resultados destacables. Ude y Coker (2012) recomiendan que el incentivo debe motivar al empleado, junto con tener un diseño flexible, buscando retroalimentación y asegurando los objetivos de desempeño esperados en la organización. Los mismos autores definieron los siguientes programas de incentivos: incentivo monetario, beneficios especiales, reconocimiento, oportunidades especiales, tasas de precios, comisiones, participación de beneficios y participación de ganancias. Ude y Coker (2012) encontraron evidencia del vínculo entre los esquemas de incentivo, motivación y productividad del trabajador, que estando bien diseñados y administrados, mejoran las organizaciones, proveyendo atractividad, motivación, desarrollo y retención de los empleados.

Según Caso (2003) uno de los beneficios de implementar un sistema de incentivos se encuentra en el aumento de la productividad, el cual, dependiendo de los niveles de rendimiento en empresas sin sistemas de incentivo, puede suponer un 33\% de aumento salarial, la disminución de los costos directos de personal, el control de la producción y una mejora de los métodos de trabajo. El mismo autor señala que existen dos elementos de compensación para los empleados, los relacionados con el tiempo y con la producción. Se debe pagar en base al tiempo cuando las unidades de producción sean difíciles de distinguir y medir o, cuando los trabajadores no pueden controlar la cantidad de producción o los retrasos en el trabajo son frecuentes y están fuera del control de los operarios o las consideraciones de calidad son particularmente importantes y cuando el conocimiento preciso de los costos de mano de obra por unidad no sea un requerimiento producto de las condiciones competitivas (Caso 2003).

Aguiar et al. (2012) evaluaron incentivos laborales para mejorar la productividad y calidad de servicios del rubro farmacias, aplicando un plan Scalon con la participación de empleados y clientes. Se propuso un modelo mixto de incentivos, tanto remunerado vinculado al nivel de ventas, como no remunerado, obteniéndose resultados significativos. Kaufman (1992) evaluó los efectos en la productividad aplicando Improshare, mostrando un aumento significativo de la productividad, en una media del $8 \%$ en el primer año y acumulativamente llega al 17,5\% en el tercero. Sin embargo, el autor indica que las tecnologías de manufactura al estar cada vez más integradas y ser más complejas, dificultan la identificación exacta del efecto obtenido por cada trabajador, tanto en cantidad como en calidad, además de que se podrían incrementar los desechos en el esfuerzo por aumentar la producción individual, o bien provocando defectos con la necesidad de rehacer el trabajo.

Bryson (1999) encontró que los esquemas de participación financiera tienen efectos significativos en empresas de gran tamaño, al contrario que en las más pequeñas. Leonard (1990) señala que los incentivos de largo plazo en las empresas generan grandes incrementos en los retornos y la equidad, por sobre aquellas que no tienen este tipo de planes.

Van y Minh (2012) estudiaron a través de encuestas a más de 240 ejecutivos jefes de pequeñas y medianas empresas, las prácticas en recursos humanos y la productividad, entre ellos el incentivo al desempeño productivo, encontrando una relación positiva entre los incentivos por desempeño y los resultados de las empresas, acotando que éste debiera estar acompañado de entrenamiento y valoración del desempeño. Luthans (2000) estudia el reconocimiento al desempeño del trabajo, concluyendo que los empleados dan alta valoración al reconocimiento del trabajo y es una efectiva herramienta de liderazgo, que se basa en el esfuerzo por contribuir al logro de los objetivos de la organización, incluso cuando acarrea leves costos o bien no se contempla la implementación de incentivos monetarios.

Arthur y Jelf (1999) aplicaron análisis de regresión para analizar los cambios longitudinales relacionados con el efecto de la participación de utilidades por parte de los trabajadores, vinculando los resultados con la tasa de ausentismo. Se obtienen resultados significativos en el largo plazo, haciendo esta práctica importante para las relaciones laborales. Los autores señalan que la situación alcanzada, podría revertirse rápidamente cuando los incentivos desaparecen, con el riesgo de volver a la condición inicial (Arthur y Jelf 1999). 
Se detecta, además, que la industria maderera no ha sido descrita suficientemente en los términos que este trabajo presenta, destacando lo realizado por McKay (1986) donde describe los programas de gainsharing utilizados en la industria de productos forestales. En este tipo de industria uno de sus aspectos sustantivos es la planificación (Del Solar et al. 2008), así como el estímulo al recurso humano, por ello el estudio de herramientas que fomenten la productividad y por ende la competitividad del sector manufacturero forestal, contribuyen a los actuales desafíos de la industria por reclutar y mantener personal calificado, con la opción de aumentar el salario de sus trabajadores y obtener un retorno debido a la eficiencia en sus procesos.

Este trabajo tuvo como objetivo general evaluar la respuesta de la productividad que se logra con la implementación de un incentivo económico en trabajadores de una empresa maderera y como específicos medir los cambios de la productividad a partir de un modelo de aplicación de incentivos que fomente la productividad y comprobar si la introducción de planes de incentivos grupales incrementa la productividad empresarial.

\section{MATERIALES Y MÉTODOS}

La unidad de estudio fue una empresa de remanufactura de madera ubicada en la Región del Maule, Chile, con una producción aproximada de 78 mil metros cúbicos al año de molduras y marcos de puertas de la especie Pinus radiata (D. Don), las que son destinadas al mercado nacional e internacional. La empresa opera en base a 25 días laborales promedio en dos turnos, cuyo proceso productivo se muestra en la figura 1. El insumo principal son blocks libres de nudos de largos variables, que son procesados en finger joint, transformados en blanks de largos fijos y predeterminados, materia prima para las máquinas moldureras.

En agosto del año 2010, la empresa decidió implementar una política para mejorar la productividad, entregando un incentivo monetario variable en función de la producción física y tiempo de producción. El modelo fue del tipo Improshare, basado en un sistema grupal que bonifica la diferencia entre el output del período y un escenario base (Figura 2).

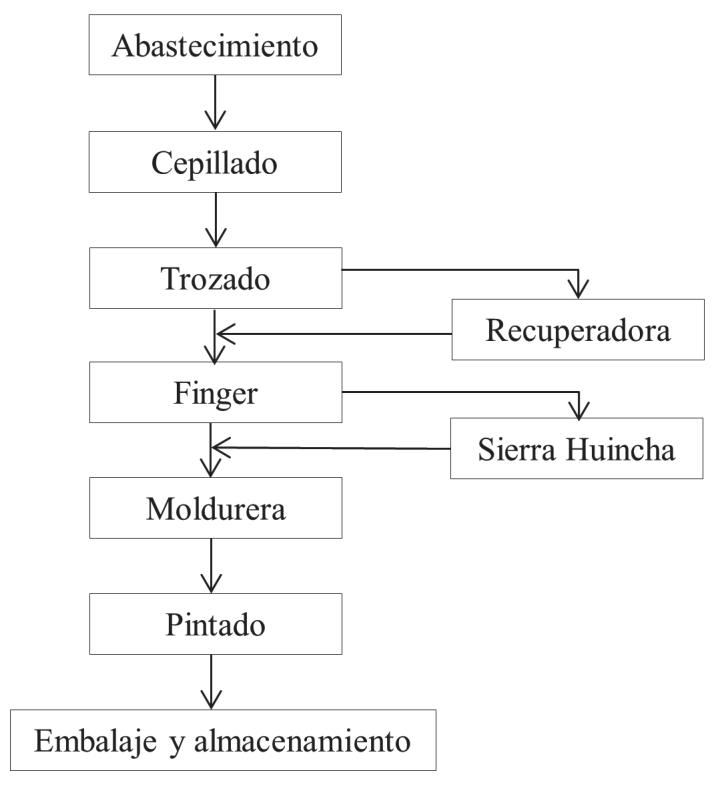

Figura 1. Secuencia del proceso productivo de la empresa. 


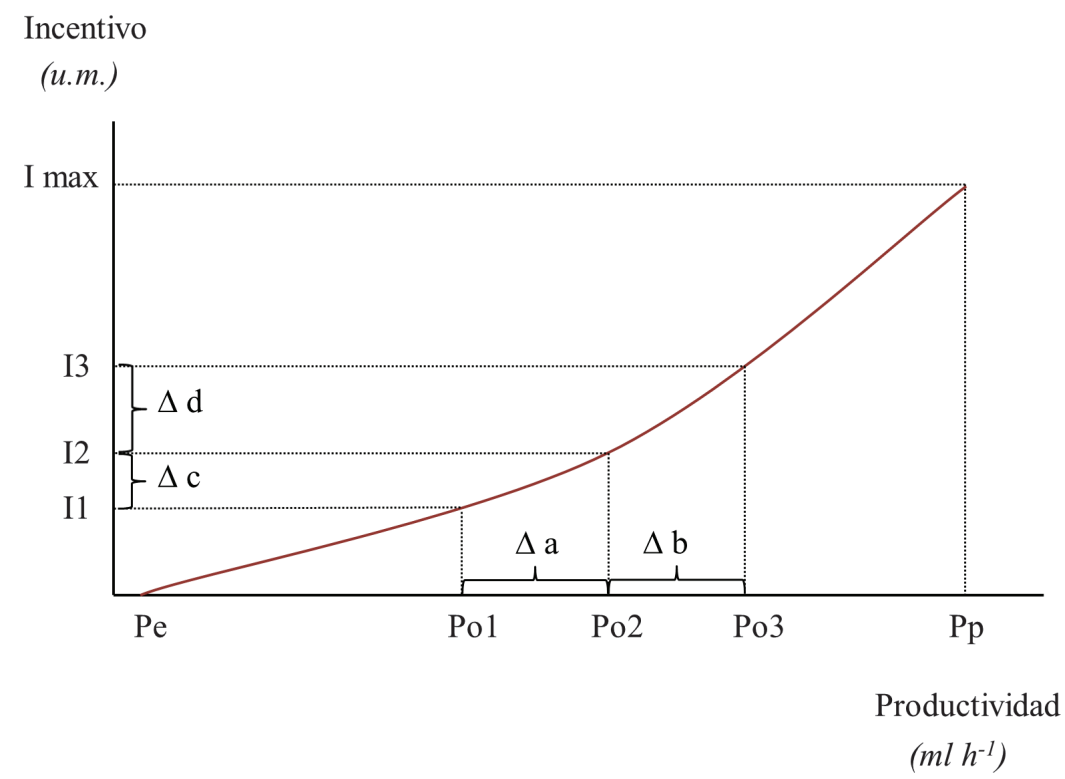

Figura 2. Evolución del incentivo a medida que cambia la productividad.

El modelo matemático de optimización implícito maximiza la siguiente función $\theta$ :

$$
\operatorname{Max} \theta=\sum_{m=1}^{j} V_{m t}-\sum_{m=1}^{j} C_{m t}-\sum_{m=1}^{j} I_{m t}
$$

Dónde:

$\theta \quad$ = función de beneficio de la empresa

$\mathrm{V}_{\mathrm{mt}}$ = ingreso por metro lineal $(\mathrm{ml})$ producidos durante el período $(t)$

$\mathrm{C}_{\mathrm{mt}}^{\mathrm{mt}}=$ costo fijo mano de obra por metro lineal $(\mathrm{ml})$ producidos durante el período $(t)$

$\mathrm{I}_{\mathrm{mt}} \quad=$ costo de la política de incentivos por metro lineal $(\mathrm{ml})$ producidos durante el período $(t)$

$\mathrm{j} \quad=\mathrm{j}$-ésima unidad producida

La implementación consideró las siguientes restricciones:

a) Si la productividad final es menor que la mínima exigible, el incentivo es cero.

b) Existe una máxima capacidad de producción definida por la capacidad nominal de producción de las máquinas.

c) Se debe mantener la calidad de la producción.

d) No debe aumentar la accidentabilidad.

e) Los tiempos muertos ocurridos por causas ajenas a la gestión de quienes laboran en la empresa no deben ser considerados. 
El modelo del incentivo se compone de una función exponencial con una constante. La primera es la diferencia de productividad entre el resultado real y la línea base. La constante es el valor del incentivo según la productividad obtenida en unidades monetarias (u.m.), equivalente al ingreso monetario mensual del puesto de ayudante.

$$
I=\left(A^{\left(P_{o}-P_{\theta}\right)}-1\right) \times K
$$

Donde:

I = incentivo

A = remuneración que aumenta según la productividad. El valor debe ser mayor a uno, en este caso $\mathrm{A}=1,001$.

Po = productividad obtenida al final de un período de evaluación.

$$
\mathrm{Po}=\frac{\mathrm{L}}{\mathrm{HC}}
$$

$\mathrm{L} \quad=$ suma de metros lineales $(\mathrm{ml})$ producidos de $(n)$ productos en $(m)$ máquinas del periodo $(i)$.

$$
\mathrm{L}=\sum_{\mathrm{i}=1}^{\mathrm{n}}\left(m l_{\mathrm{mi}}\right)
$$

$\mathrm{HC}=$ horas concedidas. Horas totales del periodo $(i)$ en $(m)$ máquinas, equivalente a las horas potenciales por turno y máquina, que se estimó en 6,6 horas.

$\mathrm{Pe}=$ productividad mínima exigible donde se inicia la bonificación de acuerdo a registros históricos de productividad. La gerencia determinó que la productividad debía crecer al menos un 5\% sobre el promedio histórico del periodo enero 2009 a junio 2010 para recibir la compensación monetaria, equivalente a una productividad media mínima de 3012 metros lineales por hora $\left(\mathrm{ml} \mathrm{h}^{-1}\right)$

$\mathrm{K}=$ unidades monetarias a retribuir para un resultado obtenido.

$$
\mathrm{K}=\frac{(\alpha-\beta)}{\mathrm{A}^{\left(\mathrm{Pp}_{\mathrm{p}}-\mathrm{Pe}\right)}-1} \times \frac{\delta}{\varphi} \times \psi
$$

$\mathrm{Pp}=$ productividad potencial, equivale a la ponderación de los $\mathrm{ml}$ posibles de producir bajo condiciones ideales con un factor de eficiencia del $100 \%$, la velocidad de diseño de las máquinas y un factor de factor de utilización (FU, para este caso del 88\%), explicado por la jornada legal de trabajo de 7,5 horas, que considera un tiempo muerto, reinicios de la máquina y aseo de 0,9 horas. La velocidad de diseño es de 60; 90 y 80 metros lineales por minutos $\left(\mathrm{ml} \mathrm{min}^{-1}\right)$ en las diferentes máquinas de la línea de producción.

Por tanto las productividades potenciales (Pp) de cada máquina son:

$$
\begin{aligned}
& \mathrm{Pp}_{\mathrm{m} 1}=60 \mathrm{ml} \mathrm{min}^{-1} \times 100 \% \times 88 \%=52,8 \mathrm{ml} \mathrm{min}^{-1}=3168 \mathrm{ml} \mathrm{h}^{-1} \\
& \mathrm{Pp}_{\mathrm{m} 2}=90 \mathrm{ml} \mathrm{min}{ }^{-1} \times 100 \% \times 88 \%=79,2 \mathrm{ml} \mathrm{min}^{-1}=4752 \mathrm{ml} \mathrm{h}^{-1} \\
& \mathrm{Pp}_{\mathrm{m} 3}=80 \mathrm{ml} \mathrm{min}^{-1} \times 100 \% \times 88 \%=70,4 \mathrm{ml} \mathrm{min}^{-1}=4224 \mathrm{ml} \mathrm{h}^{-1}
\end{aligned}
$$


La productividad potencial media se expresa como el promedio de las productividades individuales, que es equivalente a 4048 metros lineales por hora.

A = costos fijos de mano de obra por $m l$ producido cuando se cumple la productividad exigible.

$\beta \quad=$ costos fijos de mano de obra por $m l$ producido cuando se cumple la productividad potencial.

$\delta \quad=$ factor de reparto del ahorro de costos fijos entre trabajador y empresa de cada $m l$ adicional producido. La empresa determinó otorgar el $80 \%$ del ahorro de costos fijos de mano de obra debido al mejoramiento de la productividad.

$\varphi \quad=$ dotación de mano de obra incluida en el sistema de incentivo.

$\Psi \quad$ = factor por cargo.

Bonificación diferenciada según la responsabilidad del trabajador, que será de 0,9 para los ayudantes, quienes están encargados de las labores de apoyo de la producción, con una experiencia promedio de 4 años y renta de 100 u.m., presenta una dotación de 114 trabajadores; 0,95 para los operadores, quienes operan máquinas de menor complejidad y reemplaza en la máquina principal a los operadores master, tienen una experiencia promedio de 4 años y una renta de 165 u.m., tienen una dotación de 65 trabajadores; y 1 para los operadores master, que operan máquinas principales y una experiencia de más de 4 años, recibe una renta de 201 u.m. y su dotación es de 86 trabajadores. Lo anterior origina una renta promedio de 149 u.m. y el costo fijo ponderado en mano de obra de todo el personal llega a 37735 unidades monetarias.

Para analizar el impacto en la renta de los trabajadores, se analizó su variación media variable para el grupo de trabajadores, medida como u.m. Como el incentivo se relaciona con los metros lineales y el tiempo de elaboración del producto, en el resultado no influyen las variaciones de producción producto de menos días laborales o debido a interrupciones de la producción por eventos ajenos a los trabajadores, como un corte de energía. En estos casos, el impacto económico de este tipo de situaciones es absorbido por la administración.

Para analizar la productividad se usaron 19 meses antes de implementar la política de incentivos y 21 meses después de aplicar el incentivo. Para demostrar si existe diferencia se aplicó la prueba F, cuya prueba de hipótesis fue la siguiente:

$\mathrm{H}_{0}$ : La productividad no varió entre períodos con y sin incentivo.

$\mathrm{H}_{1}$ : La productividad varía entre los periodos con y sin incentivo.

El análisis de varianza paramétrico considera los supuestos de normalidad y homocedasticidad de los residuos, los cuales se comprobaron con la prueba de Shapiro-Wilk y Levene's, respectivamente. Si alguno de los supuestos no fuera aceptado, entonces se debe realizar la prueba de Kruskal-Wallis que corresponde a un análisis de varianza no paramétrico entre las medias del ranking. Los valores originales se transforman a un ranking dependiendo de su posición en el ordenamiento ascendente de los datos. Si el análisis de varianza paramétrico o no paramétrico detectó diferencias estadísticamente significativas se aplicó la prueba de rangos múltiples SNK para identificarlas. El análisis de datos se llevó a cabo con el software estadístico Statgraphics Centurion XVI.

Finalmente, para analizar el impacto de la política de incentivo sobre los resultados de la empresa, se analizó la capacidad de utilización de los equipos y el costo unitario medio de la mano de obra por metro lineal, para períodos con y sin incentivo. 


\section{RESULTADOS Y DISCUSIÓN}

\section{Análisis de Productividades entre Períodos con y sin Incentivo}

El supuesto de normalidad no se aceptó (Probabilidad $<0,01$ ); mientras que la homocedasticidad (Probabilidad $>0,05$ ) fue aceptada. Como uno de los supuestos no fue aceptado, se utilizó un análisis de varianza no paramétrico. La prueba de Kruskal-Wallis (Tabla 1), que evidenció diferencias estadísticas altamente significativas entre los periodos (Probabilidad $<0,01$ ). El periodo con y sin incentivo alcanzó un rango promedio de 29,5 y 10,6, respectivamente (diferencia del 278,3\%).

Tabla 1. Prueba de Kruskal-Wallis y de rangos múltiples SNK.

\begin{tabular}{cccc}
\hline Tratamiento & $\begin{array}{c}\text { Tamaño } \\
\text { Muestra }\end{array}$ & $\begin{array}{c}\text { Rango } \\
\text { Promedio }\end{array}$ & $\begin{array}{c}\text { Grupos } \\
\text { Homogéneos }(*)\end{array}$ \\
\hline Con incentivo & 21 & 29,4762 & $\mathrm{~A}$ \\
Sin incentivo & 19 & 10,5789 & $\mathrm{~B}$ \\
\hline
\end{tabular}

Estadístico $=26,0644$ Probabilidad $=3,30223 \mathrm{E}-7$

(*) Letras desiguales indican diferencias significativas (Probabilidad $<0,05$ ).

\section{Análisis de Rentabilidad del Sistema de Incentivo}

Como se comprobó que hubo diferencia significativa de la productividad en la implementación de una política de incentivo, a continuación se presenta el beneficio generado para verificar la rentabilidad para la empresa y para el trabajador, los resultados obtenidos muestran concordancia con lo reportado por Kaufman (1992) y Aguiar et al. (2012).

\section{Beneficio económico percibido por el trabajador}

La figura 3 muestra el incentivo en u.m. y en el recuadro variación porcentual que recibió cada trabajador $\left(\mathrm{I}_{1}\right.$, $\mathrm{I}_{2}, \mathrm{I}_{3}$ ) según cargo, en porcentaje de acuerdo a la función de la productividad del período. Se aprecia similitud en el monto del incentivo por cargo, cuya diferencia no es mayor a 0,0112 u.m., es decir, no más del 1,12\%, resultados que muestran que el incentivo dio resultado positivos (Kohn 1993, Luthans 2000, Caso 2003).

En la figura 3 se aprecia la evolución de la productividad real, incluyendo la productividad exigible (Pe) y potencial (Pp), como piso y techo, observándose un crecimiento de la productividad. El factor de utilización para períodos sin incentivo promedió $70,95 \%$, con una desviación de $3,25 \%$; en cambio, en períodos con incentivo promedió un 78,03\%; con una desviación de 2,56\%, lo que mostró un aumento de la capacidad de utilización, donde el valor más alto se obtuvo en abril de 2012 con un 82,39\%. Al respecto Suñé et al. (2004) indican que capacidad y productividad deben mantener un equilibrio adecuado, puesto que son fundamentales para tener capacidad suficiente para satisfacer la demanda y mantener un costo competitivo. 


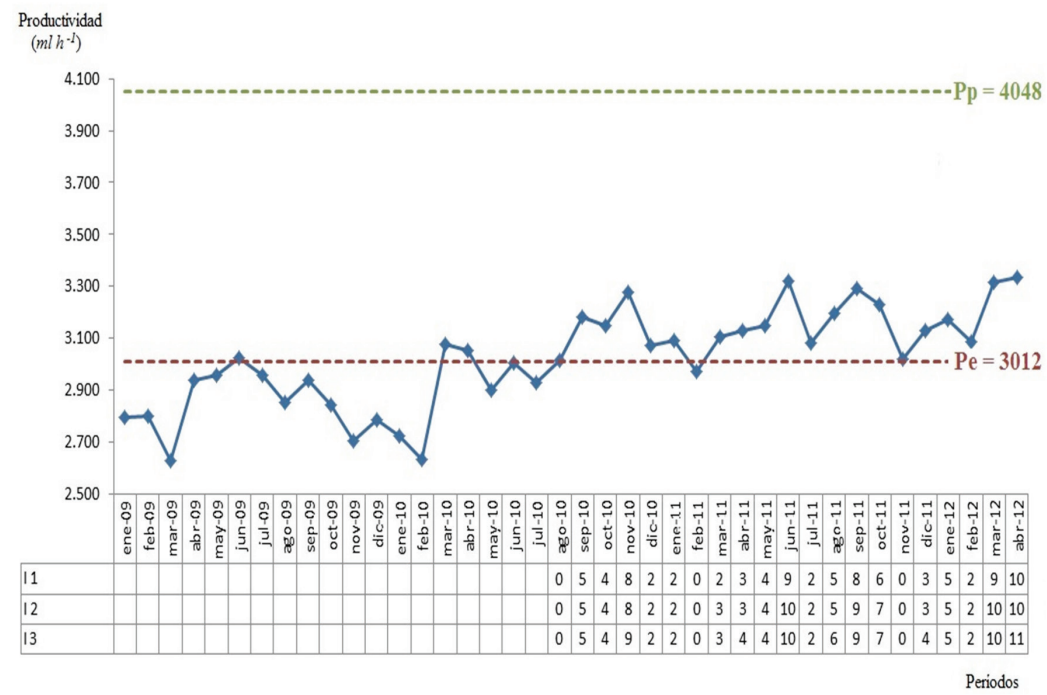

Figura 3. Productividad media e incentivo porcentual por cargo.

El incentivo medio de los tres tipos de operario fue positivo en el $86 \%$ de las ocasiones posibles (18 de los 21 meses donde se aplicó el incentivo), cuyo monto promedio máximo alcanzó el $10 \%$ de una u.m. en el mes de abril de 2012 (Figura 3).

\section{Análisis de costos}

Se evaluó si el beneficio empresarial obtenido al implementar una política de incentivo fue suficiente como para tolerar el gasto adicional que genera su uso. La figura 4 presenta la evolución del Costo Medio (CM) y Costo Medio Equivalente (CME) en base a valores constantes del mes de abril 2012, en base a 25 días hábiles del periodo, que no incluyen inflación u otra variable que la renta del trabajador. Fue observable un desacoplamiento entre CM y CME, debido a las distintas cantidades de metros lineales producidos por el número de días disponibles, situación diferente en los meses de febrero y marzo de 2010, cuyos datos son afectados por el terremoto del 27 de febrero de 2010.

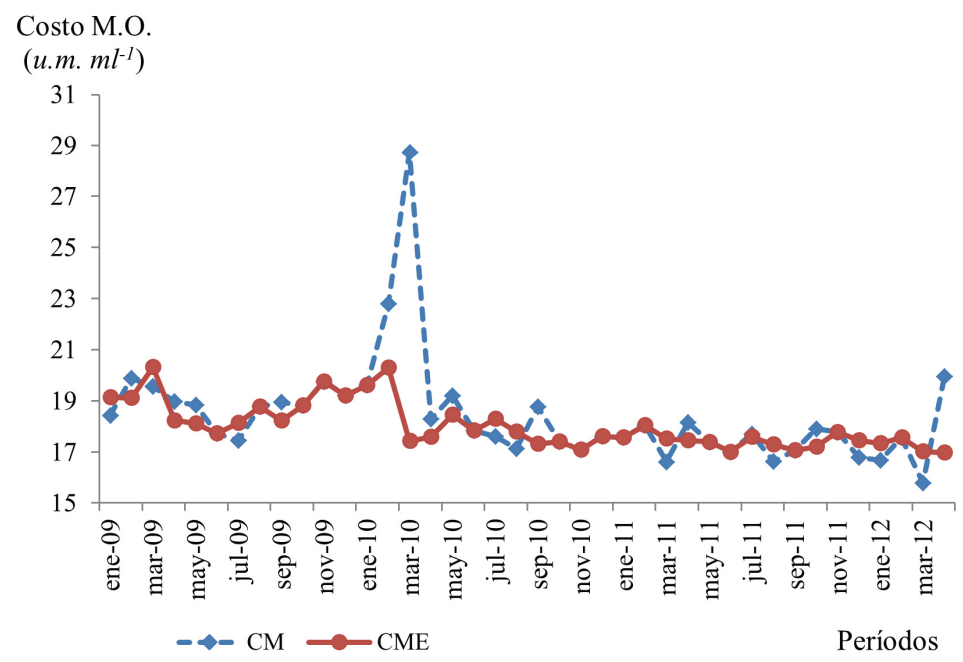

Figura 4. Costo medio (CM) y equivalente (CME) de la mano de obra real por período (millones de u.m.). 
Debido al aumento de las remuneraciones, creció el gasto total en mano de obra por período, pero se evidencia que el costo por metro lineal disminuye en el tiempo, producto de la mejora de la productividad (Figura 5).

La productividad mínima corresponde al mes de marzo del año 2009 (Figura 3 y Figura 5) período en el que no se contaba con una política de incentivo, siendo el costo de mano de obra un gasto fijo en relación a los metros lineales producidos. Situación similar ocurre en el caso de la productividad base o exigible (Pe). Por otro lado, la productividad máxima (Pmax), se logra en el mes de abril del año 2012, que incluyó el gasto adicional debido a la aplicación de la política de incentivo. Asimismo, el costo estimado para la productividad potencial $(\mathrm{Pp})$ corresponde al máximo monto de incentivo en relación a los metros lineales declarados posibles de producir.

No obstante, se revisó la magnitud de la disminución de costos, comparando el CME con la situación hipotética de CM sin política de incentivo (CMS). En la Figura 5 se observa que la curva del CMS muestra una diferencia del $25 \%$ del costo de la mano de obra al aumentar la productividad de Pe a Pp; en cambio, al analizar la curva del CME con incentivo salarial, el ahorro disminuyó un 6\%, ya que la fracción de ahorro restante se asignó como bonificación. El CME aumentó un 5,7\% respecto al CMS en el período con mayor productividad; sin embargo, si se compara con Pe el costo de la mano de obra disminuyó un 4,6\%, demostrando que el CM de la mano de obra después de implementar la política de incentivo es menor que cuando no existía política, cuyo ahorro de costos fijos de mano de obra fue repartido entre la empresa y destinado al incentivo para el trabajador, como lo señala Robbins (2004).

Los resultados, producto de la implementación de un sistema Improshare son los esperables, ya que la motivación del personal (Meyers 2000, Rynes et al. 2004, Milkovich et al. 2011) bajo un esquema flexible de remuneraciones (Ude y Coker 2012) que permite alcanzar los logros esperables, en esquemas donde trabajadores y empresa obtienen beneficios mutuos. La brecha aún por cerrar obedecería al ajuste que hay entre una planificación en base a resultados esperables nominales y reales.

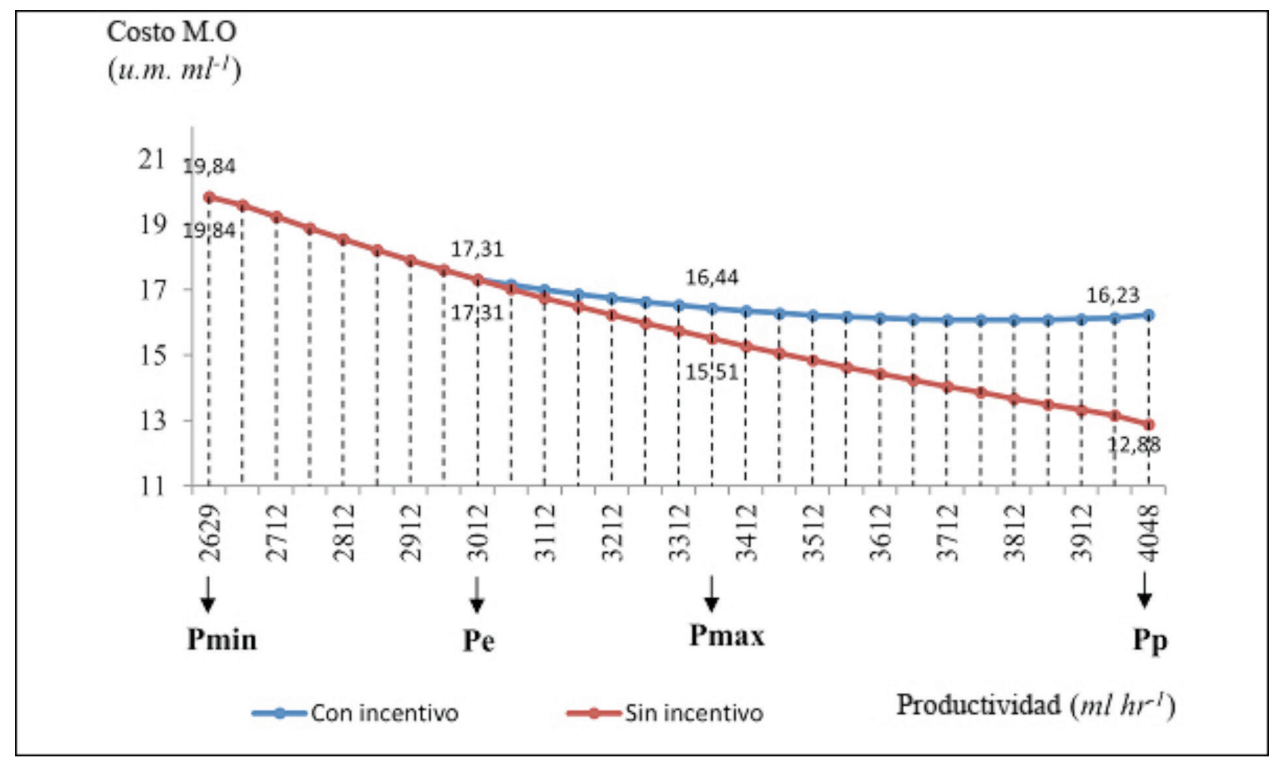

Figura 5. Costo medio de la mano de obra con y sin incentivo (millones de u.m.). 


\section{CONCLUSIONES}

La implementación de un incentivo monetario al trabajador en una empresa maderera, en base a una fracción del ahorro en costos fijos de mano de obra, producto del incremento en la producción de molduras en relación al tiempo empleado, mostró incrementos estadísticamente significativos de productividad, mejorando la renta del trabajador y la utilización de la capacidad instalada.

Los principales cambios observados fueron: la productividad llegó a crecer un $10,7 \%$ con respecto a la base establecida y un 4,8\% como promedio. La renta variable promedio mensual del trabajador llegó a representar un $10 \%$ de un sueldo mínimo.

La política de incentivo demostró ser un instrumento que estimula la productividad, asignando los recursos económicos que derivan de un mejor desempeño hacia el trabajador. A cambio, la empresa obtuvo beneficios en la reducción de costos laborales y un mayor aprovechamiento de la capacidad instalada, siendo en definitiva más competitiva que antes.

Se corroboró que a través de un incentivo en función de los resultados operativos, puede crecer el salario del trabajador sin que el incremento mismo obedezca a efectos inflacionarios.

\section{BIBLIOGRAFÍA}

Aguiar, M.; Pérez, D.F.; Madriz, R.D. 2012. Incentivos laborales como aporte a la productividad y a la calidad de servicios en las empresas de rubro farmacias. Ingeniería Industrial Actualidad y Nuevas Tendencias 3(9):33-48.

Annamalai, T.; Abdullah, A.G.K.; Alasidiyeen, N.J. 2010.The Mediating Effects of Perceived Organizational Support on the Relationships between Organizational Justice, Trust and Performance Appraisal in Malaysian Secondary Schools. European Journal of Social Sciences 13(4): 623-632.

Arthur, J.B.; Jelf, G.S. 1999. The effects of gainsharing on grievance rates and absenteeism over time. Journal of Labor Research 20(1):133-145.

Boothby, D.; Dufour, A.; Tang, J. 2010. Technology adoption, training and productivity performance. Research Policy 39(5):650-661.

Bryson, A. 1999. The impact of employee involvement on small firm's financial performance. Journal of the National Institute of Economic Research 169(1):78-95.

Buller, P.F.; Mc Evoy, G.M. 2012. Strategy, human resources management and performance: Sharpening line of sight. Human Resources Management Review 22(1):43-56.

Caso, A. 2003. Sistemas de incentivos a la producción. Fundación Confemetal. $1^{\text {a }}$ Edición. España.

Del Solar S.R.; Chacón C. I.; Ponce D. M. 2008. Plan agregado de producción en barracas madereras. Estudio de caso para una pequeña industria. Maderas: ciencia y tecnología 10(2):77-92.

Infante, C. 2008. Manual de gestión de recursos humanos III. $3^{\text {a }}$ Edición. Fondo Editorial UNET. Venezuela. 
Kamalian, A.R.; Yaghoubi, N.M.; Moloudi, J. 2010. Survey of Relationship between Organizational Justice and Empowerment (A Case Study). European Journal of Economics, Finance and Administrative Sciences 24: 165-171.

Kaufman, R. 1992. The effects of Improshare on productivity. Industrial and Labor Relations Review 45(2):311-322.

Kohn, A. 1993. Why incentive plans cannot work. Harvard Business Review 71(5): 54.

Leonard, J.S. 1990. Executive pay and firma performance. Industrial and Labor Relations Review 43(3):13-29.

Luthans, K. 2000. Recognition: a powerful, but often overlooked, leadership tool to improve employee performance. The Journal of Leadership Studies 1(7):31-39.

Meyers, F. 2000. Sistemas de incentivos y comisiones. En: Estudios de tiempo y movimiento manufactura ágil. $2^{\mathrm{a}}$ Edición. México.

McKay, M.B. 1986. Company/Employee gainsharing programs. Studies in management and accounting for the forest products industries. Oregon State University, School of Business. USA.

Milkovich, G.; Newman, J.; Gerhart, B. 2011. Compensation. 11a Edition. McGraw Hill/Irwin. United States.

Rynes, S.L.; Gerhart, B.; Minette, K.A. 2004. The importance of pay in employee motivation: discrepancies between what people say and what they do. Human Resource Management 43(4):381-394.

Robbins, S. 2004. Programas de pago variable. En: Comportamiento organizacional. 10ª Edición. Pearson Educación. México.

Segura, R. 2011. La pyme maderera en cifras. Concepción, Chile. [Consultado: 07.06. 2012]. [Disponible en] <http://web.ffm.cl/attachments/article/55/lapymeforestalmaderera.pdf.>

Suñé, A.; Gil, F.; Arcusa, I. 2004. Diseño de procesos de producción. En: Manual práctico de diseño de sistemas productivos. $1^{a}$ Ed. Díaz de Santos. España.

Ude, U.; Coker, M.A. 2012. Incentive scheme, employee motivation and productivity in organizations in Nigeria: Analytical Linkages. Journal of Business and Management 1(4):32- 39.

Van, T.N.; Ninh, N.N. 2012. Effects of human resources management on business performance of small and medium size manufactures in Hanoi - Vietnam. Australian Journal of Business and Management Research 2(6):47-54.

Vergara, M. 1998. Productividad, salarios y negociación colectiva. Temas Laborales $\mathrm{N}^{\circ} 10$. [Consultado: 27.12.2012]. [Disponible en] <http://www.dt.gob.cl/documentacion/1612/w3-article-60343.html>.

Yazdani, B.O.; Yaghoubi, N.M.; Giri, E.S. 2011. Factors affecting the empowerment of employees. European Journal of Social Sciences 20(2): 267-274. 